\title{
Electronic health records contributing to physician burnout
}

- Cite as: CMAJ 2017 November 13;189:E1405-6. doi: 10.1503/cmaj.109-5522

Posted on cmajnews.com on Oct. 26, 2017.

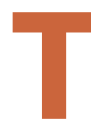
hough touted as a vital component of modern health care, the electronic health record (EHR) is having an unfortunate effect on many physicians. The clerical burden introduced by EHRs has become a leading cause of physician burnout.

"EHRs contribute to burnout by turning physicians into unhappy data-entry clerks, and also by enabling 24-hour patient access without any system to provide compensation or coverage," said Dr. Robert Wachter, professor and chair of the department of medicine at the University of California, San Francisco.

Wachter was a plenary speaker at the 2017 American Conference on Physician Health, held recently in San Francisco. His spoke about how EHRs contribute to physician burnout, and what changes need to occur to address this problem.

The downside of the widespread adoption of EHRs (also known as EMRs, short for electronic medical records) is increasingly of interest to the physician wellness community. A 2016 survey of 6375 doctors in the United States, published in Mayo Clinic Proceedings, found that $84.5 \%$ of them used EHRs. Physicians who used EHRs reported feeling less satisfied with the amount of time they spent on clerical tasks. They were also found to be at higher risk of professional burnout.

A smaller study from 2014, involving 370 primary care physicians and 92 clinic managers in New York City, had similar findings. Physicians who reported moderate use of EHRs were less satisfied with their jobs and had higher levels of stress. Those who fell into the high-use category reported feeling greater time pressures, which was "associated with significantly more burnout, dissatisfaction and intent to leave" the medical profession.

According to research conducted by the RAND Corporation, a nonprofit think tank, senior physicians in particular loathe the "cumbersome, time-consuming data entry" that comes with using EHRs. Other problems expressed to RAND by doctors

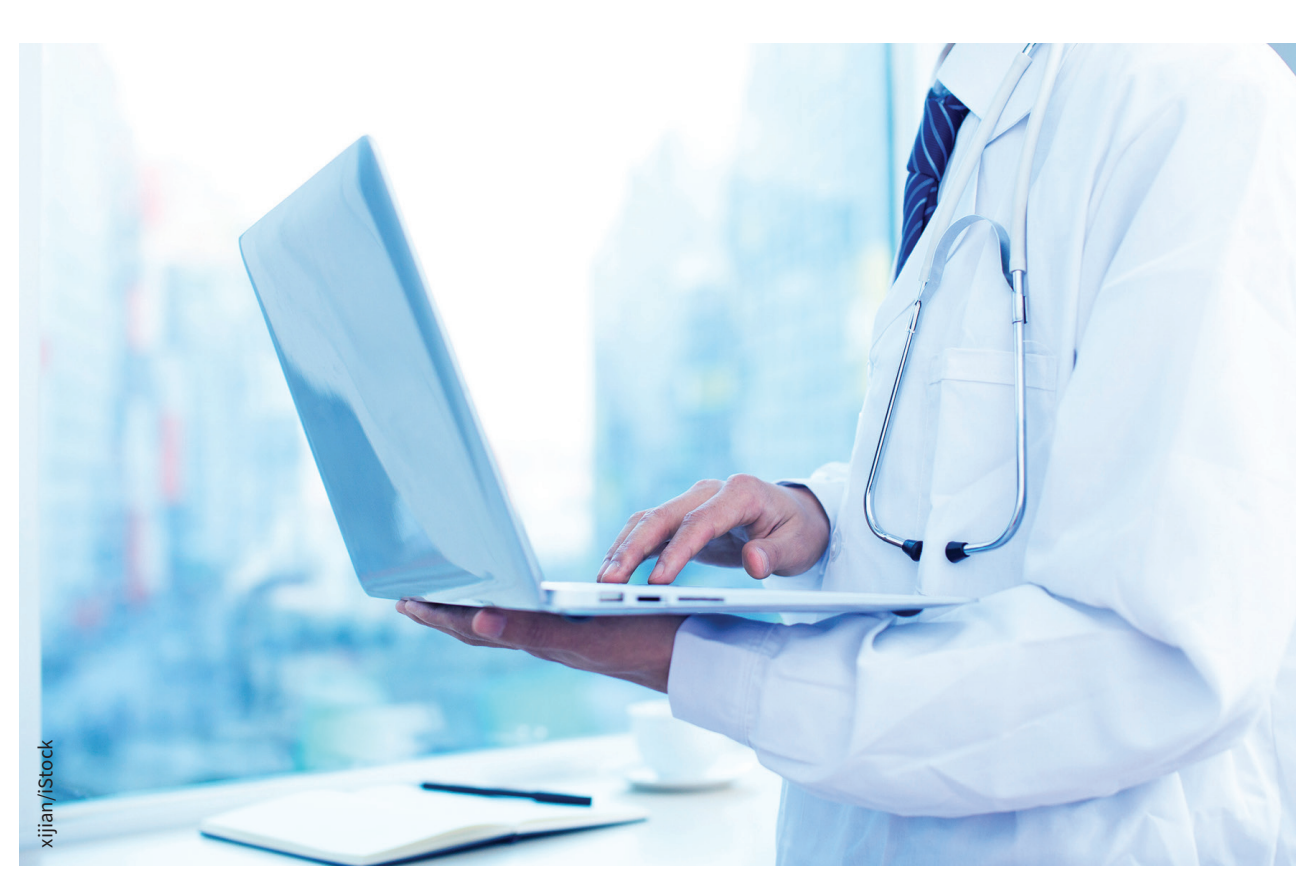

Many physicians aren't happy about how much time they spend entering data into electronic health records.

included nonintuitive user interfaces, information overload, lower-quality documentation resulting from template-based notes, and the lack of interoperability between electronic systems.

Some doctors have reported working longer hours because of EHRs, many completing their clerical work at home during evenings or weekends. The systems are also designed to make billing easier, not to make clinical care more efficient, others have noted - so tasks such as refilling prescriptions actually take longer then before EHRs.

Of greater concern to many physicians is that more facetime with screens means less connection with patients and fewer discussions with colleagues. "We sit sideby-side doing data entry and interacting

our machines without interacting with each other," one physician lamented about how EHRs have changed the practice of medicine.

Even critics of EHRs, however, realize the potential of technology to improve clinical practice, but they suggest the technology has to improve. This will involve having EHR vendors working more closely with doctors to make the systems more clinically relevant. User interfaces 
need to improve, and how EHRs function must align better with physician workflows in clinical settings.

In the future, artificial intelligence and machine learning may help EHRs anticipate much of the work now performed by hand so that many tasks could be automated. How doctors practise medicine may also have to evolve to match the progress made possible by advancements in medical technology.

"There are two keys to improving the situation with EHRs. The first is to build better systems - which may be done by the existing EHR companies or by new tools and apps developed by others," said Wachter. "The second is to reimagine the work - to think of new ways of providing care that take advantage of the technology without slavishly replicating the old paper-based work and workflow."

Roger Collier, CMAJ 